\title{
RECENT DEVELOPMENTS IN LASER-DRIVEN POLARIZED SOURCES
}

\author{
L. Young, K. P. Coulter, R. J. Holt, E. R. Kinney, \\ R. S. Kowalczyk, D. H. Potterveld, A. Zghiche \\ Physics Division \\ Argonne National Laboratory \\ Argonne, IL 60439 USA \\ CONF-900924--11 \\ DE9 1006600
}

\begin{abstract}
Recent progress in the performance of laser-driven sources of polarized hydrogen and deuterium is described. The current status of the prototype source, $I=2.5 \times 10^{17} \mathrm{~s}^{-1}$, polarization $=0.29$ (including atomic fraction), is comparable to classical Stern-Gerlach sources. A scheme to improve source performance by approximately an order of magnitude, using a combination of optical-pumping spin-exchange and RF transitions, is outlined.
\end{abstract}

\section{INTRODUCTION}

The development of high-density targets of polarized hydrogen and deuterium is central to the study of spin-dependent structure in nuelei. While such targets are of general interest, two experiments in particular motivate our development of polarized internal gas targets of hydrogen and deuterium: a) measurement of tensor analyzing power in electron-deuteron elastic scattering [1] and b) ineasurement of spin-dependent structure functions of the neutron and proton [2] . In both experiments, the desired figure of merit for the target is $n p_{z z}^{2} \approx n p_{z}^{2} \geq 10^{14}$ nuclei $/ \mathrm{cm}^{2}$. In ordex to achieve this thickness, the proposed scheme is to feed a windowless storage cell [3] by a polarized source. At present, two options for the polarized source are being actively pursued, namely, the Stern-Gerlach source [4] at Heidelberg and the laser-driven source at Argonne [5].

The laser-driven polarized source is based on the principle of spin-exchange optical pumping. In this method, optical pumping creates a spin-polarized alkali atom, which, via spin-exchange collisions will polarize the electron of the hydrogen or deuterium atom. The nucleus of the $\mathrm{H}$ or $\mathrm{D}$ atom becomes polarized at low magnetic fields via the hyperfine interaction. This technique has great potential, which can be understood by considering that the photon flux availahle from a standara Ti-sapphire laser at the potassium D1 resonance line is 3-4 watts. A conversion efficiency of only $10 \%$ would give polarized $\mathrm{H} / \mathrm{D}$ atoms at a flux $\geq 10^{18} \mathrm{~s}^{-1}$, far exceeding the flux available from conventional sources, $3 \times 10^{16} \mathrm{~s}^{-1}$. In this paper, recent developments (using both potassium and sodium as the spin-exchange intermediate) which show the laser-driven source to be comparable to the benchmark Stern-Gerlach source [6] will be discussed.

\section{EXPERIMENTAL}

\section{MASTER}

The prototype spin-exchange optical pumping system is shown schematically in Figure 1. $\mathrm{An} \mathrm{Ar}^{+}$-pumped dye laser system provides the radiation required to optically pump the alkali, either $\mathrm{Na}$ or $\mathrm{K}$. The spectral density of the laser is tailored to match 
the Doppler-broadened absorption profile of the alkali vapor, circularly I larized, and sent to the spin-exchange cell located near the center of the diagram.

The spir-exchange cell is constructed of pyrex as an integral unit with the rfdissociator and alkali reservoir. The interior is coated with drifilm [7]. The entire cell is heated to $\sim 250^{\circ} \mathrm{C}$ to prevent alkali condensation and placed in a static holding field of $10 \mathrm{G}$. The $H / D$ flux is controlled and measured with a servo-driven needle valve. The alkali density is independently controlled by the reservoir temperature and quantified by measuring the integrated absorption coefficient with a probe laser. Typically the ratio of $N$ is to $D$ atoms is 0.1 to $0.3 \%$. The mixture of alkali and $D$ atoms exits the spinexchange cell through a spout, after which a chopper and quadrupole mass spectrometer are nsed to determine the fraction of $\mathrm{D}$ atoms in atomic form (AF).

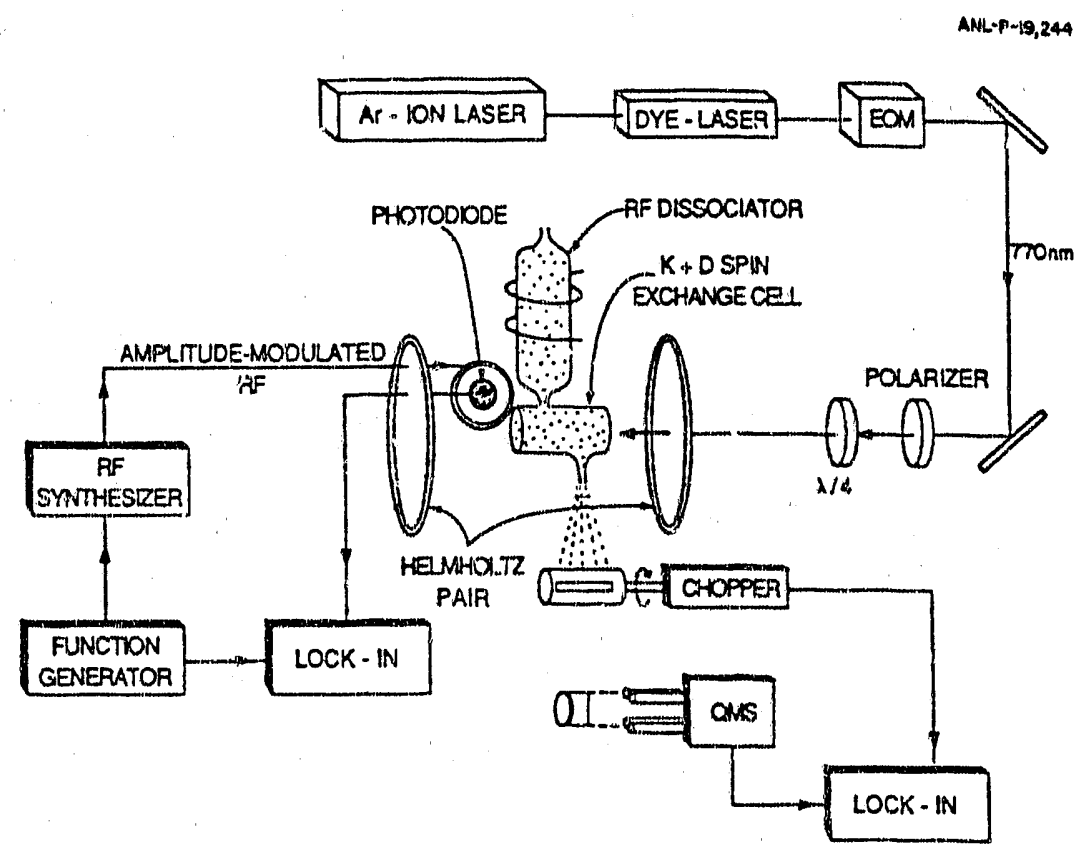

Figure 1. Schematic of the laser-driven source.

The equilibrium polarization of the atoms is measured by optical detection of magnetic resonance transitions between adjacent Zeeman sublevels [8]. That is, increased fluorescence from the optically-pumped alkali atoms is observed as a result of altering the relative populations of the magnetic sublevels, either directly or via spin-exchange collisions with deuterium atoms. A typical signal using the Zeeman technique is shown in Fig. 2. The asymmetry between $\sigma+$ and $\sigma$ - pumping reverses as expected. The polarizations shown on the figure are deduced using a spin-temperature model, where the populations of the magnetic sublevels are given by $N_{m,} \propto e^{\beta m_{f}}$, where $\beta$ is the spin temperature. From the figure, there appears to be an incomplete equilibration between the polarization of the alkali and deuterium atoms.

The average deuterium polarization is expected to be measured well, since, the mean free path between $D$-alkali collisions is much larger inan the cell dimensions $(\lambda=$ 
$\left.1 / \sigma_{\text {se }} \mathrm{n}_{\mathrm{A}} \approx 10^{3} \mathrm{~cm}\right)$. However, the alkali polarization may be overestimated, since regions of the cell with high laser intensity are preferentialiy sampled. This is confirmed by probe laser measurements of differential $\sigma+, \sigma-$ absorption as a function of position in the cell. The calculated polarization transfer efficiency is thus an underestimate, and improvements in deuterium polarization can be expected with uniform high-power laser coverage of the cell.

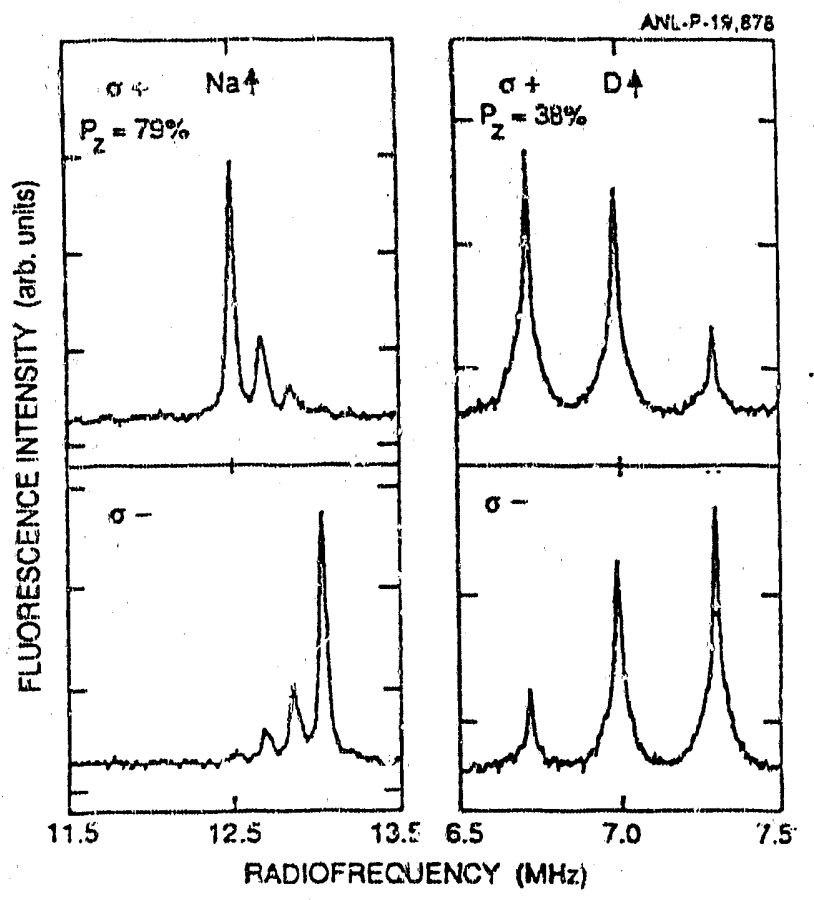

Figure 2. Typical fluorescence signal using Zeeman technique.

\section{RESULTS AND DISCUSSION}

In this section we discuss the evolution of the laser-driven source. Only deuterium will be discussed in detail, although tests have shown that hydrogen polarization essentially equivalent to deuterium vector polarization, $p_{z}$, can be obtained. Iritial studies (ANL'88) were conducted using potassium as the spin-exchange iutermediate. This choice was motivated by the ease with which one could distribute the output of a $\mathrm{cw}$ laser over the absorption profie of the $\mathrm{K}$ vapor $(\approx 1.5 \mathrm{GHz}$ Doppler + hyperfine) relative to other alkalis. The switch to $\mathrm{Na}$ (ANL'90) as the spin-exchange intermediate can be best understood by considering the processes which determine the deuterium polarization.

The processes in the spin-exchange cell can be modelled using a ratic equation approach, which leads to the following relationship for the efficiency of polarization transfer from tine alkali to the $D$ atom.

$$
P_{D}=\frac{\gamma_{e e} P_{A}}{\gamma_{\rho e}+\Gamma}\left(1-e^{-\left(\gamma_{\iota e}+\Gamma\right) t}\right)
$$


where $\gamma_{s *}=$ spin-exchange rate $=\sigma_{s e} \bar{v} n_{A}, \Gamma=$ loss rate (depolarization + flow rate) and $P_{D}, P_{A}=$ electron polarization of the deuterium and alkali, respectively.

In order to enhance the efficiency of polarization transfer, one should maximize the spin-exchange rate, i.e. $n_{A}$, and minimize the loss rate. However, due to radiation trapping, $n_{A}$ can not be increased arbitrarily without a corresponding decrease in $P_{A}$. Radiation trapping at low $B$-fields is a less severe problem for $\mathrm{Na}$ than for $\mathrm{K}$. The critical density (where the alkali polarization is degraded by roughly $50 \%$ ) is more than a factor of two higher for $\mathrm{Na}$ than for $\mathrm{K}$.

Table 1 shows the performance figures for the laser-driven source configurations at low fields using $\mathrm{K}$ (ANL'88) and $\mathrm{Na}$ (ANL'90) as the spin-exchange intermediates. In addition, it shows the corresponding parameters for the benchmark classical atomic beam source (Bonn). The first three columns concentrate solely on source performance $p_{z}$, I and atomic fraction (AF). The final two columns relate to the expected figure of merit $\left(F=I p_{z}^{2} A F^{2} \epsilon\right)$ for internal target applications involving a storage cell, as indicated in the introduction. The injection efficiency, $\epsilon$, into the storage cell (fourth column) is unity for the laser-drivin source since the source and cell can be directly coupled. The figure of 0.5 given for the Bonn source is estimated from the experience of the ANL-Novosibirsk collaboration [1]. It may be an underestimate, but is included to indicate the non-trivial nature of efficient injection into the storage cell.

Table I. Performance figures for polarized H/D sources.

\begin{tabular}{cccccl}
\hline Source & $\mathrm{p}_{z}$ & $\begin{array}{c}\text { Intensity } \\
\left(\times 10^{17} s^{-1}\right)\end{array}$ & $\begin{array}{c}\text { Atornic } \\
\text { Fraction }\end{array}$ & $\begin{array}{c}\text { Irjection } \\
\text { Efficiency }\end{array}$ & $\begin{array}{l}F \\
\left(s^{-1}\right)\end{array}$ \\
\hline$A N L^{\prime} 88$ & 0.25 & 0.6 & 0.80 & 1.0 & $0.24 \times 10^{16}$ \\
$A N L^{\prime} 90$ & 0.38 & 2.5 & 0.75 & 1.0 & $2.0 \times 10^{16}$ \\
Bonn & 0.95 & 0.3 & 1.00 & 0.5 & $1.4 \times 10^{16}$ \\
\hline
\end{tabular}

As can be seen, the switch from potassium to sodium increased the deuterium polarization, $p_{z}$, from 0.25 to 0.38 , for the reasons previously discussed. Another very marked improvement is the source intensity. This is due primarily to the increased laser power. The incident photon fluxes for the $\mathrm{K}$ and $\mathrm{Na}$ cases were $\approx 8 \times 10^{17} \mathrm{~s}^{-1}$ and $\approx 3 \times 10^{18} \mathrm{~s}^{-1}$, respectively.

\section{FUTURE PLANS}

Although substantial gains have been posted with the change in alkali and additional laser power, the laser-driven source has not yet met the design goals: I = $4 \times 10^{17} s^{-1}, p=0.5$. In particular, the $p_{x z}$ obtained thus far, 0.15 , yields $n$ figure of merit similar to the existing source at Novosibir $\}$ and must be improved an order of rnagnitude for the electron-deuteron elastic scattering experiments.

In order to increase the polarization $\left(p_{z z}\right)$ and the intensity of the source, we plan a two-step process: optical-pumping spin-exchange with $\mathrm{K}$ at high field followed by $\mathrm{RF}$ transitions in a moderate field. The added complication of RF transitions subsequert to the spin-exchange is offset by the advantages of high-field optical pumping, namely, greatly reduced radiation trapping problems and better wall depolarization properties. The $p_{x z}$ expected in this scheme is $p_{z z}=\epsilon_{24} A F P_{D}$, where $\epsilon_{24}$ is the efficiency of the $4 \leftrightarrow 3$ and $3 \leftrightarrow 2$ RF transitions. Monte Carlo simulation of the process gives $\epsilon_{24} \approx 0.9$. 
Based on previous experience, we estimate a $P_{D}=0.7$ and $A F=0.8$ to be achievable, leading to a $\left|p_{z z}\right|=0.50$. A recently-acquired Ti-sapphire laser provides an increase of a factor of 4 in available photon flux $\left(1.2 \times 10^{19} \mathrm{~s}^{-1}\right)$. Thus, deuterium atom fluxes of $\sim 1 \times 10^{18} \mathrm{~s}^{-1}$ should be achievable.

\section{SUMMARY}

Over the past two years, an increase of approximatily a factor of 8 in figure of merit has been realized for the laser-driven source of polarized deuterinm. The gains have been due to a change in the spin-exchange intermediate from potissium to sodium and increased laser power. In its present state, $I p_{2}^{2} A F^{3}=2.0 \times 10^{16} \overrightarrow{\mathrm{D}} / \mathrm{s}$ the laser-driven source is already comparable to classical atomic beam sources. Further improvements (a factor of $\sim 12$ ) are expected with increased laser power and a new scheme employing high-field optical-pumping spin-exchange followed by RF transitions.

\section{ACKNOWLEDGEMENTS}

We thank J. Gregar for his state-of-the-ait glassblowing, as well as C. Kurtz and R. Amrein for technical assistance. Finally, we thank D. K. Toporkov and D. M. Nikolenko for assistance during some of the experimexts, and R. A. Gilman for helpful discussions. This work was supported by the U. S. Department of Energy, Nuclear Physics Division, under Contract No. W-31-109-ENG-38.

\section{REFERENCES}

[1] R. A. Gilmain et al., Phys. Rev. Lett. 65, 1733 (1990).

[2] "A Proposal to Measure the Desp Inelastic: Spin Dependent Structure Functions of the Nucleon at HERA", the HERMES collaboration, DESY-PRC-90-01 (1990).

[3] Barker, M. D. et al., in Polarization Phenomera in Nuclear Physics - 1980, edited by G. G. Ohlsen et al., AIP Conference Proceedings No. 69 (American Institute of Physics 1981), p. 931.

[4] G. Graw et al., in Proceedings of the IV LEAR Workshop, Villars, 1987, ed. C. Amsler et al., Harwood Acad. Publ., Chur (1988) p. 249.

[5] L. Young et al., in Proceedings of the lopical Conference on Electronucleax Physics with Internal Targets, Stanford, CA, 9-12 January 1989, ed. R. Arnold (World Scientific, Singapore) p. 125; Nucl. Phys. A497, 529c, (1989).

[6] H. G. Mathews et al., Nucl. Instru. and Meth. 213, 155 (1983).

[7] L. Young et al., Nucl. Instru and Meth. B24/25, 963 (1987); D. R. Swenson and L. W. Anderson, Nucl. Instru. and Meth. B29, 627 (1988).

[8] W. E. Bell and A. L. Bloom, Phys. Rev. 107,1559 (1957); S. G. Redsun et al.,Phys. Rev. A42, 1293 (1990). 





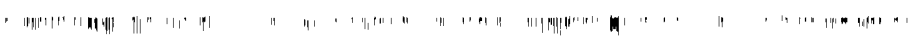

